\title{
Uma Avaliação Sistemática de Aprendizagem e Usabilidade para o TuPy Online
}

\author{
Jorge Luiz de Jesus Goulart, UERJ, jorge_goulart@outlook.com, \\ https://orcid.org/ 0000-0001-6605-1917 \\ Fabiano de Souza Oliveira ${ }^{1}$, UERJ, fabiano.oliveira@ime.uerj.br, \\ https://orcid.org/ 0000-0002-8498-2472 \\ Paulo Eustáquio Duarte Pinto ${ }^{2}$, UERJ, pauloedp@ime.uer.br, \\ https://orcid.org/ 0000-0002-7393-3464 \\ Antônio Orestes de Salvo Castro, UERJ, orestes@ime.uerj.br, \\ https://orcid.org/ 0000-0002-4973-9995
}

Resumo: Este estudo teve como objetivo avaliar a usabilidade e a eficácia da ferramenta TuPy Online na aprendizagem de disciplinas do curso de Ciência da Computação da Universidade do Estado do Rio de Janeiro. O TuPy Online foi idealizado como uma ferramenta para visualização de execução de algoritmos, com ênfase na exibição de estruturas de dados, em diferentes níveis de abstração. Utilizando a metodologia de Savi et al. para avaliação de aprendizagem, verificamos que a introdução da ferramenta produziu impacto positivo e, considerando o aspecto de percepção dos alunos, um impacto ainda mais expressivo. Medimos a usabilidade usando o questionário SUS, além de um questionário em busca de um retorno específico referente às funcionalidades do TuPy Online. Os resultados foram positivos e propiciaram o mapeamento de possíveis melhorias do software.

Palavras-chave: aprendizagem. algoritmos. TuPy Online. estatística não-paramétrica.

\section{Usability Assessment and Algorithm Learning Using TuPy Online}

Abstract: This study aimed to evaluate the usability and effectiveness of the TuPy Online tool in learning subjects in the Computer Science course at the State University of Rio de Janeiro. TuPy Online was conceived as a tool for visualizing the execution of algorithms, with an emphasis on displaying data structures at different levels of abstraction. Using the methodology of Savi et al. for learning assessment, we found that the introduction of the tool had a positive impact and, considering the students' perception aspect, an even more significant impact. We measured usability using the SUS questionnaire, in addition to a questionnaire for a specific feedback regarding the features of TuPy Online. The results were positive and provided the mapping of possible improvements to the software.

Keywords: learning. algorithms. TuPy Online. nonparametric statistics.

\section{Introdução}

Nos últimos tempos, tem sido notória a migração de métodos tradicionais de ensino para métodos alternativos. Com o grande avanço tecnológico e a popularidade da Internet, ambientes digitais dão oportunidade a uma nova forma de aprendizagem, tornando-se frequente o desenvolvimento de softwares projetados para o complemento do processo de ensino. Em 2018, um grupo de pesquisadores da Universidade do Estado do Rio de Janeiro (UERJ), idealizou a ferramenta TuPy Online ${ }^{3}$, como um software livre, para amparar e auxiliar estudantes na aprendizagem de algoritmos, e com três objetivos

\footnotetext{
${ }^{1}$ Parcialmente financiado pela FAPERJ

${ }^{2}$ Parcialmente financiado pela FAPERJ

${ }^{3}$ Disponível em https:/gvirtu.github.io/TuPyOnline/
} 
explícitos: (i) introduzir uma pseudolinguagem com um número reduzido de palavraschave para construção de algoritmos; (ii) basear-se na língua portuguesa com comandos e identificadores escritos em português, atendendo um público-alvo sem domínio da língua inglesa; (iii) permitir ao usuário uma visualização descomplicada do progresso da estrutura de dados frente à depuração do código linha por linha.

Este trabalho tem como objetivos principais apresentar o TuPy Online (Seção 2), descrever e discutir a aplicação de uma metodologia (Seções 3 e 4) para avaliação de software educacional baseada nas contribuições de [Savi et al. 2010] e [Ferreira 2017]. Além disso, descreve, também, uma avaliação de usabilidade do TuPy Online através do questionário SUS [Brooke 1996] e de um questionário próprio como feedback para possíveis melhorias do TuPy Online. Por fim, apresentam-se os resultados na Seção 5 e a conclusão deste trabalho na Seção 6.

\section{TuPy Online}

A ferramenta possui quatro componentes principais: OPT, Graphviz, ANTLR4 e Interpretador para TuPy. O OPT ${ }^{4}$ é uma ferramenta de apoio ao aprendizado de Python e utilizada em inúmeras universidades em todo o mundo. O Graphviz é um pacote de código aberto dedicado a representação de informação estruturada na forma de grafos e redes. Foi criado por pesquisadores da AT\&T Labs em 1991 e possui apoio da comunidade até hoje. O ANTLR4 é um gerador de analisadores sintáticos a partir de definições de gramáticas. É, por exemplo, usado no mecanismo de buscas do Twitter. Finalmente, o interpretador da linguagem TuPy foi desenvolvido em Python e é responsável pelo processamento do fluxo de execução do programa. Para o melhor entendimento da arquitetura, dois dos componentes são detalhados a seguir: o OPT e o interpretador da linguagem TuPy.

A interface do TuPy Online é totalmente baseada no navegador e herda diversos aspectos do OPT. Conforme mostra a Figura 1, a página principal consiste do campo de edição de texto e dos botões de ação. A parte inferior da página também conta com um manual da linguagem TuPy e uma coleção de exemplos representativos de diferentes algoritmos e estruturas de dados. Durante a visualização, um painel de código situa-se à esquerda com destaque às linhas atual e seguinte da sequência de execução, junto aos botões de navegação. Assim como no OPT, é possível configurar pontos de parada por meio de um clique na linha de código desejada. Alguns pontos de destaque implementados são a possibilidade de fornecer dados de entrada do usuário a serem lidos pelo programa, assim como botões para salvar e carregar o código-fonte de um arquivo de texto. O editor de código também foi adaptado para habilitar conveniências como preenchimento automático, expansão ou contração de blocos (code folding) e uma fonte ${ }^{5}$ com suporte a ligaduras voltadas à programação, isto é, a capacidade de unir símbolos consecutivos de um código em um novo símbolo coerente automaticamente. A digitação dos símbolos correspondentes ao comparador de não-igualdade de operandos (“! ="), por exemplo, resulta na exibição do símbolo " $\neq$ ”. Com isso, espera-se que o código TuPy possa se aproximar visualmente de notações de pseudocódigo manuscrito. Além disso, foi disponibilizada uma versão que pode ser executada localmente, sem necessidade de conexão com a internet, minimizando problemas de escalabilidade oriundos do compartilhamento de recursos do servidor.

\footnotetext{
${ }^{4}$ Disponível em http://pythontutor.com/

${ }^{5}$ Fira Code. Dispon'1vel em https://github.com/tonsky/FiraCode. Acessado em 18 fev. 2018.
} 


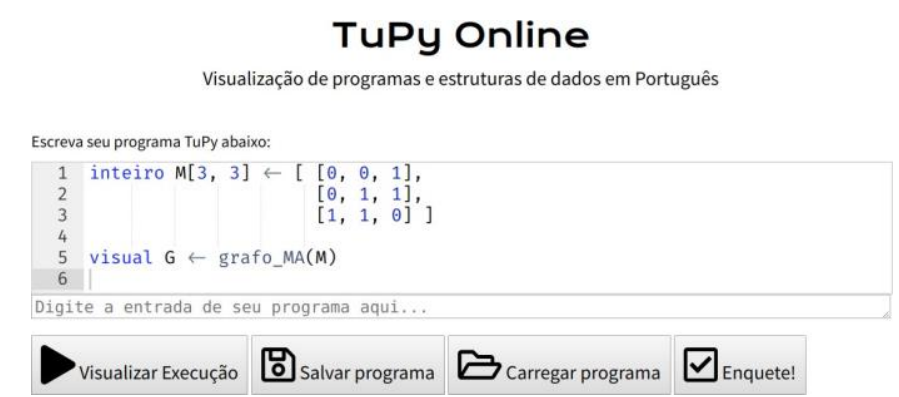

Figura 1. A interface de edição de código do TuPy Online.

\subsection{Visualização de Estruturas de Dados}

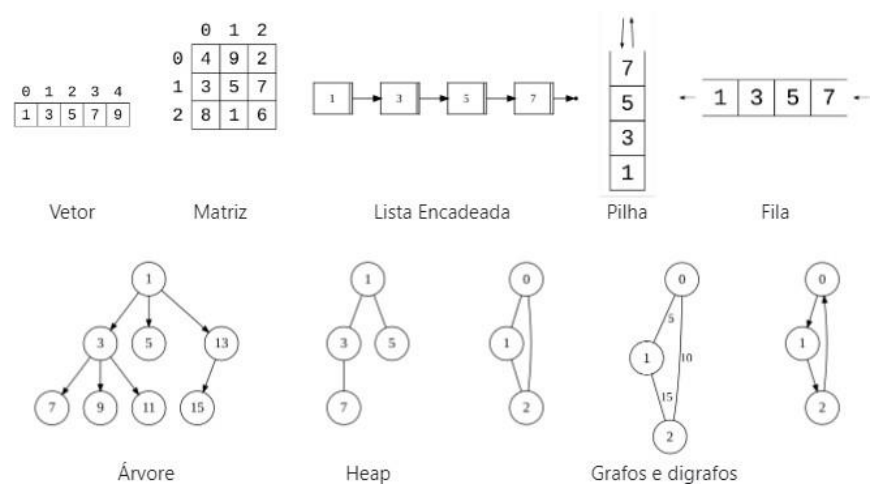

Figura 2. Exemplos de visualizações para estruturas de dados no Tupy Online

O projeto do TuPy Online providenciou uma biblioteca de funções para criar representações visuais extensíveis a partir de estruturas de dados. A Figura 2 mostra as principais visualizações já prontas para diversas estruturas de dados: vetores, matrizes, listas encadeadas, pilhas, filas, árvores enraizadas, grafos e digrafos com ou sem pesos. Internamente, as funções encapsulam lógica para percorrer as estruturas convertendoas para descrições textuais, que são consumidas e transformadas em imagens durante a execução no navegador do usuário pelo Graphviz, uma ferramenta para visualização de grafos e produção de diagramas estáticos descritos pela linguagem DOT.

Visualizações específicas também podem ser criadas, como é o exemplo da Figura 3. Isto pode ser feito com o uso da linguagem DOT, que possui uma extensa gama de opções para a customização dos formatos, das cores e do posicionamento dos elementos visuais, o que permite gerar visualizações parametrizadas pelo estado do programa. Isso significa que, além das funções predefinidas para estruturas de dados convencionais, o programador pode criar novas funções capazes de exibir imagens com as características que desejar, complementando a visualização de forma integrada. Essa funcionalidade é observada na demonstração do problema das $N$ rainhas em um tabuleiro de xadrez, incluído como um dos exemplos da ferramenta para ilustrar a customização da visualização para problemas específicos.

\section{Exemplo da proximidade da visualização com a abstração da estrutura de dados}

Considere o problema da obtenção de uma Árvore Geradora Mínima (AGM) em um grafo com pesos. Essa é uma importante aplicação de grafos na interconexão de redes, pois uma AGM indica a forma mais barata de se obter interconexão dos diversos 


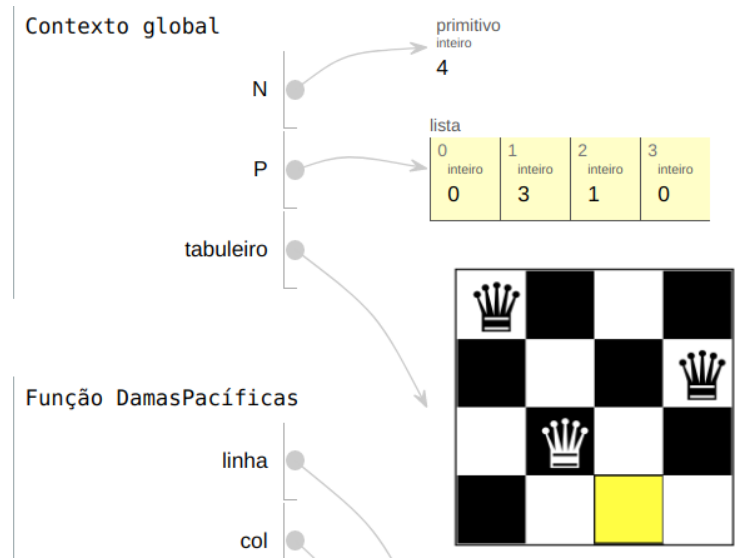

Figura 3. Tabuleiro de xadrez gerado por uma função TuPy durante a execução do algoritmo de backtracking para o problema $N$ Rainhas $(\mathrm{N}=4)$.

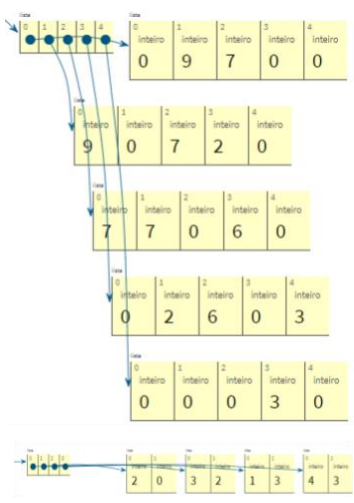

(a)

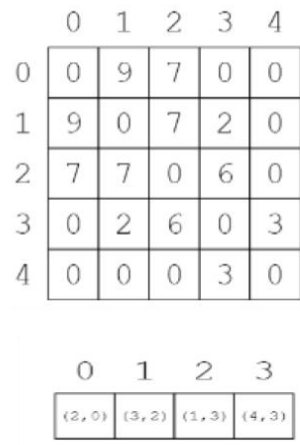

(b)

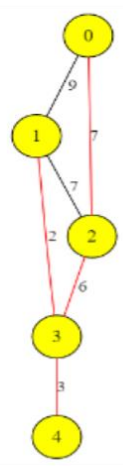

(c)

Figura 4. Representações em forma de listas (a), matrizes/vetores (b) e grafo (c).

nós de uma rede, modelada como um grafo com pesos. A Figura 4 ilustra três diferentes visualizações de uma AGM, obtida pelo algoritmo de Prim. Esse é um algoritmo guloso que constrói a AGM passo a passo, iniciando com um vértice qualquer. A cada passo, um novo vértice e uma nova aresta do grafo original são escolhidos e integrados à árvore em construção. A aresta escolhida deve ser aquela de custo mínimo, sujeita à restrição de que um dos vértices incidentes já esteja na árvore e o outro não. A parte esquerda da figura ilustra duas listas na memória. Aquela da parte superior contém um conjunto de listas da representação interna da matriz de adjacências do grafo. A parte inferior mostra uma lista das arestas escolhidas: $(2,0),(3,2),(1,3)$ e $(3,4)$. Essas representações são aquelas feitas pelo OPT, bastante básicas. Na parte central da figura, o grafo é exibido como uma matriz de adjacências e as arestas escolhidas como um vetor. Pode-se notar que a visualização já torna mais fácil o entendimento do resultado. Finalmente, na parte da direita, a representação é em forma gráfica usual de um grafo, muito mais próxima da abstração que normalmente se deseja sobre tal estrutura de dados. Aqui, as arestas escolhidas são coloridas de vermelho, permitindo comparar diretamente a sobreposição das duas estruturas: o grafo e a AGM. Observe que os vértices estão coloridos com amarelo. A possibilidade de colorir os vértices e as arestas permite uma melhor compreensão do desenrolar do algoritmo. A Figura 5 ilustra vários passos do algoritmo onde os vértices já escolhidos para a AGM são coloridos e os demais continuam em branco. 


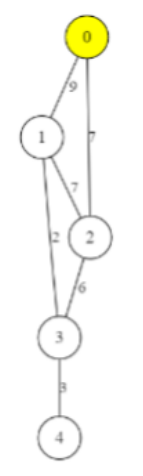

(a)

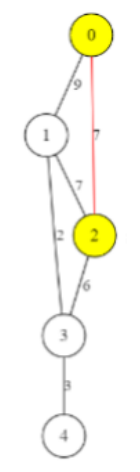

(b)

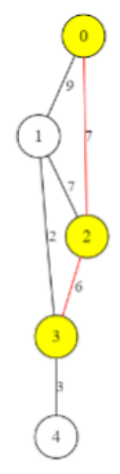

(c)

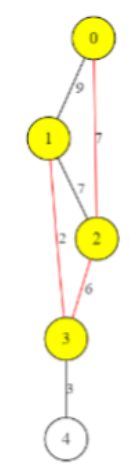

(d)

Figura 5. Visão parcial da escolha das arestas: (b): aresta $(0,2)$; (c): aresta $(2,3)$ e (d): aresta $(1,3)$.

\section{Metodologias para Avaliação de Aprendizagem e Usabilidade}

Em linhas gerais, a grande maioria das criações de modelos analíticos de aprendizagem empenha-se em tratar um problema específico, escorando todo o planejamento do experimento para atender as caracter' 1 sticas do ambiente de aprendizagem utilizado, seja ele um jogo, software educacional, ou outro método computacional.

Para avaliação do TuPy Online, foi escolhida a metodologia proposta por Savi et al. que sugere uma rápida e fácil aplicação, não exigindo competências avançadas na área de educação, medição e estatística. O questionário, chamado de $M E E G A$, apoia-se nos 4 Níveis de Kirkpatrick para aprendizado: Reação, Aprendizagem, Comportamento e Resultados. O Nível 1, Reação, identifica a percepção do aluno quanto ao conteúdo estudado. O Nível 2, avalia a mudança na forma de perceber a realidade e aumento de conhecimento e habilidades. O Nível 3, identifica a extensão da mudança de conduta que ocorre devido à participação no treinamento. Por fim, o Nível 4 avalia se o treinamento trouxe resultado/ganhos para a empresa após o aprendizado do empregado/aluno. Savi et al. utiliza-se apenas do Nível 1 e o divide em 3 partes: Motivação, Experiência e Aprendizagem. Estas 3 partes são descritas por outras metodologias de ensino: Taxonomia de Bloom [Krathwohl 2002], Modelo ARCS de Keller [Keller 1983] e Aprendizagens de Curto Prazo e Longo Prazo [Moody and Sindre 2003].

A Motivação foi dividida pelo Modelo ARCS de Keller: Atenção, Relevância, Confiança, Satisfação. A Atenção avalia se o software conseguiu ter e manter a atenção do aluno. A Relevância verificou se o conteúdo do software é importante. A Confiança verifica se o aluno obteve sensações de progresso. Por fim, a Satisfação avalia a importância e aplicação do que foi aprendido pelo aluno. A Experiência foi avaliada por Savi et al. em 5 fatores: Imersão, Diversão, Controle, Desafio e Competência. Já a Aprendizagem foi descrita pela Taxonomia de Bloom: Conhecimento, Compreensão e Aplicação do conteúdo. Além da proposta de [Moody and Sindre 2003] para avaliar a aprendizagem em um curto e um longo prazo. Além disso, optou-se por também utilizar a proposta feita por [Ferreira 2017] com o acréscimo do Nível 2 de Kirkpatrick - Aprendizagem. A autora aplicou um pré-teste antes do uso de um software e um pós-teste após a utilização do mesmo. Por fim, realizou a comparação das duas avaliações para verificar se o software beneficiou o desempenho. 
Em nenhum dos experimentos relatados foi inserido grupo de controle. Essa técnica permite o estudo experimental de um tratamento, comparando os resultados de um grupo sob o efeito do tratamento com outro grupo (grupo de controle) sem tal efeito. Optamos então, por incluir um estudo com grupos de controle.

Para a análise de usabilidade do TuPy Online, foi utilizado o questionário System Usability Scale - SUS. O SUS foi escolhido devido a número pequeno de questões e pela proposta de incluir ao questionário perguntas positivas e negativas de maneira alternada, facilitando a identificação de respostas incoerentes, o que permite a remoção de tais respostas da análise final. As 10 questões do SUS avaliam: a satisfação do usuário, a facilidade de memorização, as inconsistências, a eficiência e a facilidade de aprendizagem.

\section{Experimento}

O experimento foi realizado na Universidade do Estado do Rio de Janeiro (UERJ), com alunos da graduação em Ciência da Computação, da seguinte forma: uma turma de Otimização em Grafos (OTG), uma turma de Algoritmos e Estrutura de Dados I (AED1) e uma turma de Algoritmo e Estrutura de Dados II (AED2). Além disso, foi utilizado também em uma turma no curso de mestrado da disciplina obrigatória de Algoritmos (ALG). Os alunos estudaram durante o início do curso com a metodologia tradicional. Todos os alunos foram informados e consentiram em participar do experimento. $\mathrm{O}$ experimento transcorreu da seguinte maneira: realização do pré-teste, utilização do TuPy Online por duas semanas e, por fim, aplicação de um pós-teste, além dos questionários de percepção (Nível 1 de Kirkpatrick) e usabilidade (SUS).

O grupo de controle foi adotado para avaliar o aprendizado, mantendo o foco no uso da ferramenta, e visando excluir outras variáveis tais como: modelos de provas distintas, qualidade de ensino intrínseco a cada professor e alunos com pré-conhecimento do conteúdo da disciplina. O grupo de controle foi formulado da seguinte forma: As provas do pré-teste foram dividas nos modelos $A$ e $B$ e cada turma dividida em dois grupos de mesmo tamanho (aleatoriamente). O grupo que fez no pré-teste a prova $A$, realizou a prova $B$ no pós-teste. Já o outro, realizou o inverso, prova $B$ no pré-teste seguida da prova $A$ no pós-teste. Utilizaram-se os testes t pareado, Wilcoxon pareado e U de Mann-Whitney para as análises entre pré-teste e pós-teste, além dos grupos de controle. Os resultados são apresentados com nível de confiança de 95\%. O questionário de percepção foi elaborado com 19 questões, além de uma auto-avaliação, seguindo os princípios da Taxonomia de Bloom. Por fim, o aluno respondeu o questionário SUS para avaliar a usabilidade. Os questionários do TuPy Online estão publicamente disponíveis ${ }^{6}$.

\section{Apresentação dos Resultados}

Nas Figuras 6 e 7, apresentam-se os resultados obtidos com o questionário de percepção. A Figura 6 representa as dimensões Motivação e Experiência do Nível 1 de Kirkpatrick. Todos os critérios obtiveram pelo menos 55\% de concordância e nível de discordância abaixo dos $20 \%$, o que indica uma percepção altamente positiva em relação à ferramenta em análise.

Na dimensão Motivação, o item Relevância foi o que obteve maior avaliação. Os alunos atribuíram grande importância aos conteúdos oferecidos pelo TuPy Online. Sobre

\footnotetext{
${ }^{6}$ https://www.dropbox.com/sh/ktvsxtghv19r9ao/AACU6xOWwNFY5Y9p4B2TXgEda?dl=0/
} 


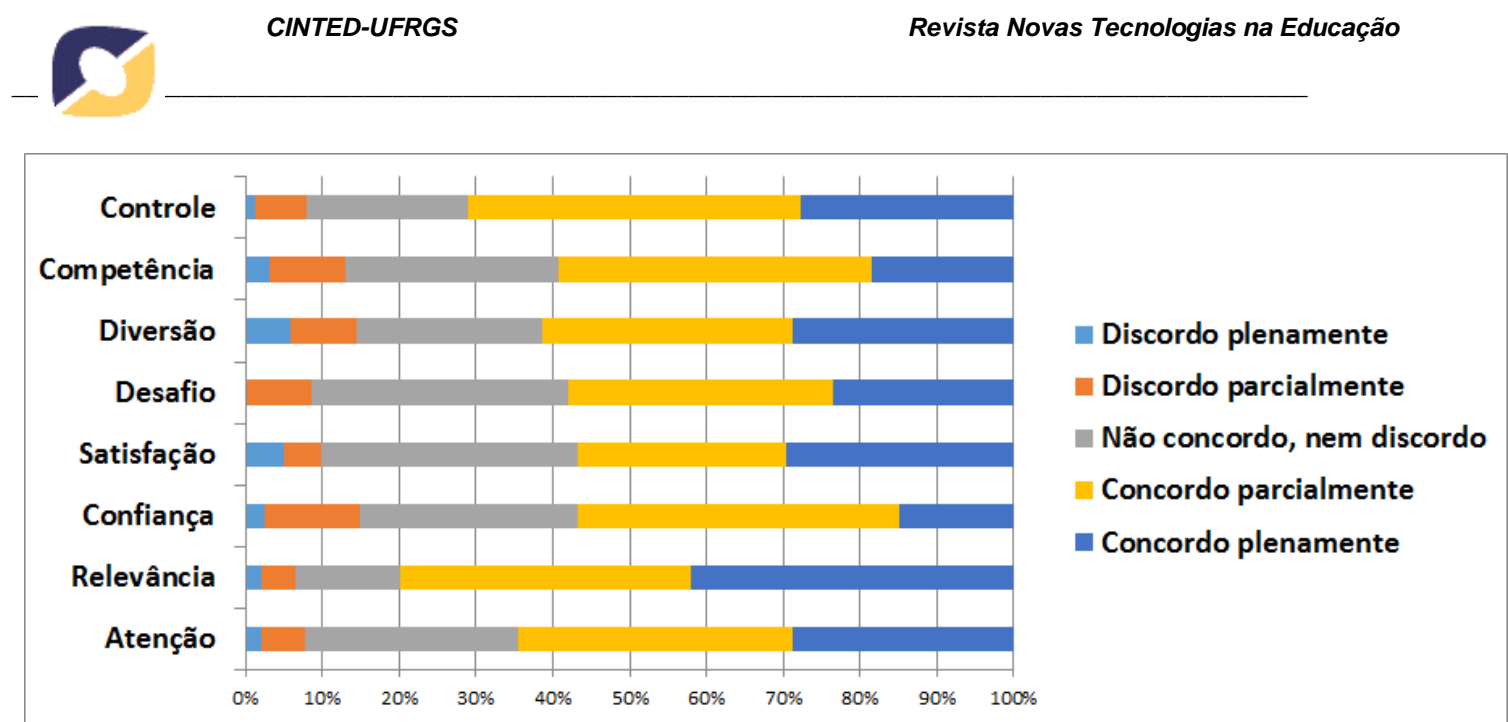

Figura 6. Avaliação do questionário de percepção.

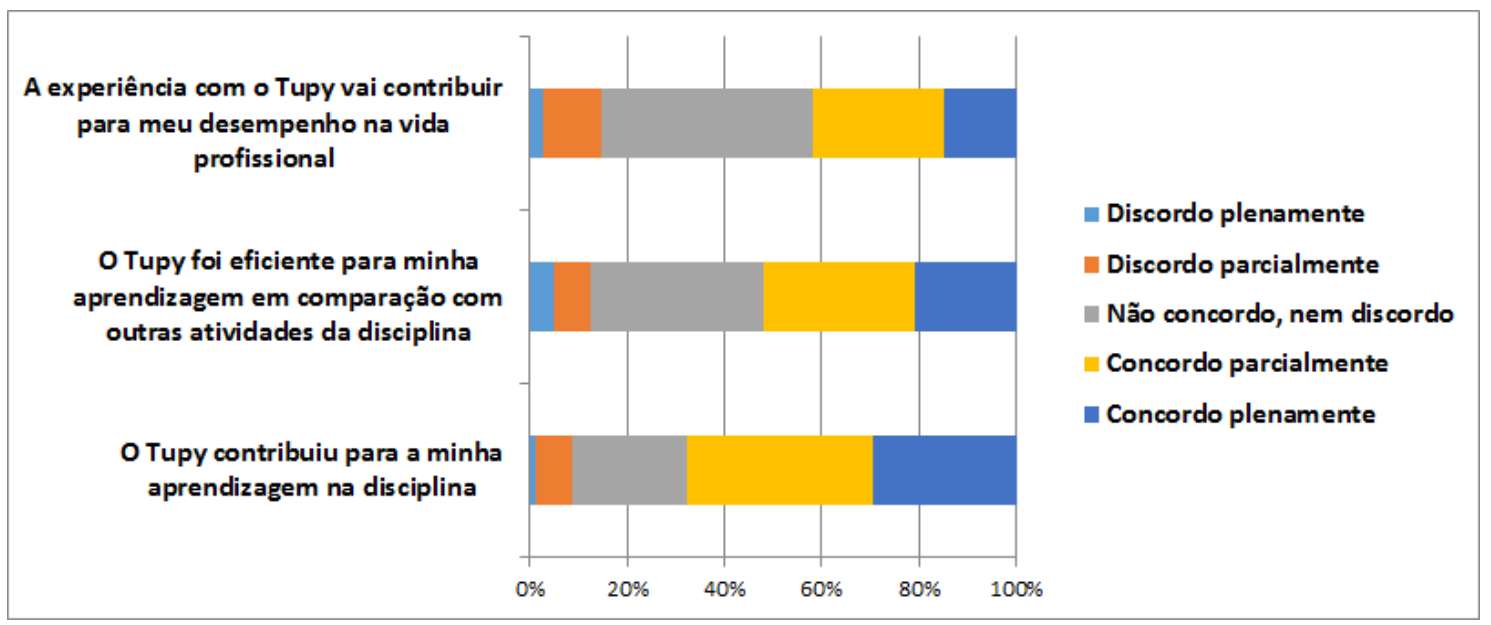

Figura 7. Aprendizagem de curto e longo prazo.

a Experiência do usuário, a dimensão Controle foi a melhor avaliada, ou seja, dada a experiência do aluno, a facilidade para usar os controles e interfaces do software, bem como a rapidez para realizar ações obtiveram destaque perante aos usuários. Em uma análise geral, todos os itens do questionário de percepção apresentaram índice com mais de $50 \%$ de concordância.

Quando questionados sobre a influência do TuPy Online na aprendizagem (Figura 7), verificou-se que os alunos acreditam que o software tem grande importância e influência para o aprendizado na disciplina. No entanto, apesar da boa avaliação, não o consideram como uma ferramenta que contribua para o desempenho profissional. Tais percepções coincidem com os objetivos do TuPy Online, em ser uma ferramenta educacional no ensino de programação.

A Tabela 1 apresenta os resultados da auto-avaliação dos alunos. Verificou-se que, para todos os assuntos abordados nas disciplinas, os alunos se auto-avaliaram melhores ao lembrar, compreender e aplicar a metodologia estudada após a utilização do TuPy Online.

$\mathrm{Na}$ avaliação de aprendizagem (Nível 2 de Kirkpatrick) pelas notas obtidas, a hipótese nula adotada foi a não influência do TuPy Online no aprendizado. Discutimos os resultados a seguir. Considerando os alunos de todas as disciplinas, em que 85 alunos 


\begin{tabular}{|c|c|c|c|c|c|c|}
\hline \multirow{2}{*}{ AED1 } & \multicolumn{2}{|c|}{ Lembrança } & \multicolumn{2}{|c|}{ Compreensão } & \multicolumn{2}{|c|}{ Aplicabilidade } \\
\hline & Antes & Depois & Antes & Depois & Antes & Depois \\
\hline Listas Lineares & 2,61 & 3,00 & 2,55 & 3,38 & 2,11 & 2,61 \\
\hline Pilhas & 3,00 & 3,44 & 2,50 & 3,22 & 2,27 & 2,72 \\
\hline Filas & 3,06 & 3,44 & 2,77 & 3,38 & 2,50 & 2,83 \\
\hline \multirow{2}{*}{ OTG } & \multicolumn{2}{|c|}{ Lembrança } & \multicolumn{2}{|c|}{ Compreensão } & \multicolumn{2}{|c|}{ Aplicabilidade } \\
\hline & Antes & Depois & Antes & Depois & Antes & Depois \\
\hline Busca em Grafos & 2,66 & 3,12 & 2,66 & 3,20 & 2,37 & 3,12 \\
\hline Ordenação Topológica/COM & 2,66 & 3,04 & 2,58 & 3,12 & 2,37 & 3,00 \\
\hline Componentes Fortemente Conexos & 2,37 & 3,04 & 2,37 & 3,08 & 2,33 & 2,95 \\
\hline \multirow{2}{*}{ AED2 } & \multicolumn{2}{|c|}{ Lembrança } & \multicolumn{2}{|c|}{ Compreensão } & \multicolumn{2}{|c|}{ Aplicabilidade } \\
\hline & Antes & Depois & Antes & Depois & Antes & Depois \\
\hline Divisão e Conquista & 3,00 & 3,55 & 2,88 & 3,77 & 2,66 & 3,33 \\
\hline Recursão e Memorização & 3,33 & 3,77 & 3,55 & 3,77 & 3,11 & 3,44 \\
\hline Programação Dinâmica & 3,44 & 3,77 & 3,55 & 3,77 & 3,11 & 3,44 \\
\hline \multirow{2}{*}{ ALG } & \multicolumn{2}{|c|}{ Lembrança } & \multicolumn{2}{|c|}{ Compreensão } & \multicolumn{2}{|c|}{ Aplicabilidade } \\
\hline & Antes & Depois & Antes & Depois & Antes & Depois \\
\hline Listas Lineares & 2,36 & 2,86 & 2,56 & 3,13 & 2,10 & 2,66 \\
\hline Pilhas & 2,53 & 3,06 & 2,60 & 3,26 & 2,33 & 2,86 \\
\hline Filas & 2,20 & 2,73 & 2,33 & 2,83 & 2,20 & 2,63 \\
\hline
\end{tabular}

Tabela 1. Resultados da avaliação pela Taxonomia de Bloom.

participaram, a análise indicou a rejeição da hipótese nula, para o p-valor de 0,0101. Portanto, houve uma melhora significativa no aprendizado dos alunos com a utilização do TuPy Online. Na análise turma por turma, obteve-se os seguintes resultados. Na disciplina AED1, nenhum dos 2 grupos rejeitou a hipótese nula. Ou seja, não foram encontradas evidências estatísticas para comprovar que o TuPy Online influenciou no desempenho dos alunos. Em OTG e AED2, apenas 1 grupo rejeitou a hipótese nula, indicando melhora no desempenho nas provas. Já na disciplina de ALG, o TuPy demonstrou diferença apenas no primeiro grupo, rejeitando a hipótese nula. No segundo grupo, rejeita-se a hipótese nula ao desconsiderarmos os alunos que reportaram não terem estudado em casa com o TuPy Online. Em relação aos grupos de controle, criados para estudar o efeito das provas no desempenho dos alunos, o resultado foi inconclusivo em mostrar que elas não tiveram influência. Sendo assim, há a possibilidade de que a melhoria observada seja parcialmente devida a provas com n'1veis de dificuldades distintas.

O resultado da análise sobre usabilidade apresenta o percentil de respostas positivas encontrado, de 66\%. Comparando com a análise feita por [Sauro 2011], o resultado é bem próximo à média de $68 \%$. Essa média está homogeneamente distribuída entre os itens analisados, sendo o de pior avaliação a Satisfação, com $60 \%$ do total de pontos possíveis e o melhor avaliado a Eficiência, com $72 \%$. Esses resultados sugerem que os usuários consideram as funcionalidades do software bem integradas, de execução rápida, mas ainda necessitam de ajuda técnica para o uso da ferramenta. Para o restante do questionário de usabilidade, criado exclusivamente para avaliação do TuPy Online os alunos avaliaram os aspectos da linguagem, interface e o processo introdutório do software.

Em relação aos aspectos de linguagem, os alunos indicaram como pontos positivos os itens: sistema intuitivo, fácil, didático e com linguagem em português, com linguagem simples, direta, possibilitando uma visualização simplificada do algoritmo e a possibilidade de executar o programa passo a passo. Em relação aos pontos negativos, os alunos indicaram a pouca explanação dentro do programa para criação de visualizações customizadas. Na avaliação sobre interface, os pontos positivos foram: interface simples, limpa, bem integrada, leve, objetiva e funcional. Já os comentários negativos referem-se ao enquadramento das visualizações na tela, botões com maior destaque na tela, problemas de edição do código após o início do algoritmo e problemas com letras maiúsculas no algo- 
ritmo. Este último problema, na versão atual do programa, já encontra-se resolvido. Por fim, o processo introdutório obteve as seguintes avaliações. Positivas: aula e explicações com clareza e objetividade em transmitir o máximo de conhecimento para uso do TuPy Online. Os pontos negativos, em geral, referem-se ao pouco tempo do experimento, a falta de exemplos mais básicos para o aprendizado da linguagem TuPy e possibilidade de um maior suporte ao programa. Além disso, a avaliação se propôs a encontrar quais foram os programas com maior número de implementações realizadas pelos alunos (Figura 8), com o intuito de perceber a necessidade de utilizar a visualização da estrutura de dados ou do processo do algoritmo através das visualizações customizadas do TuPy Online.

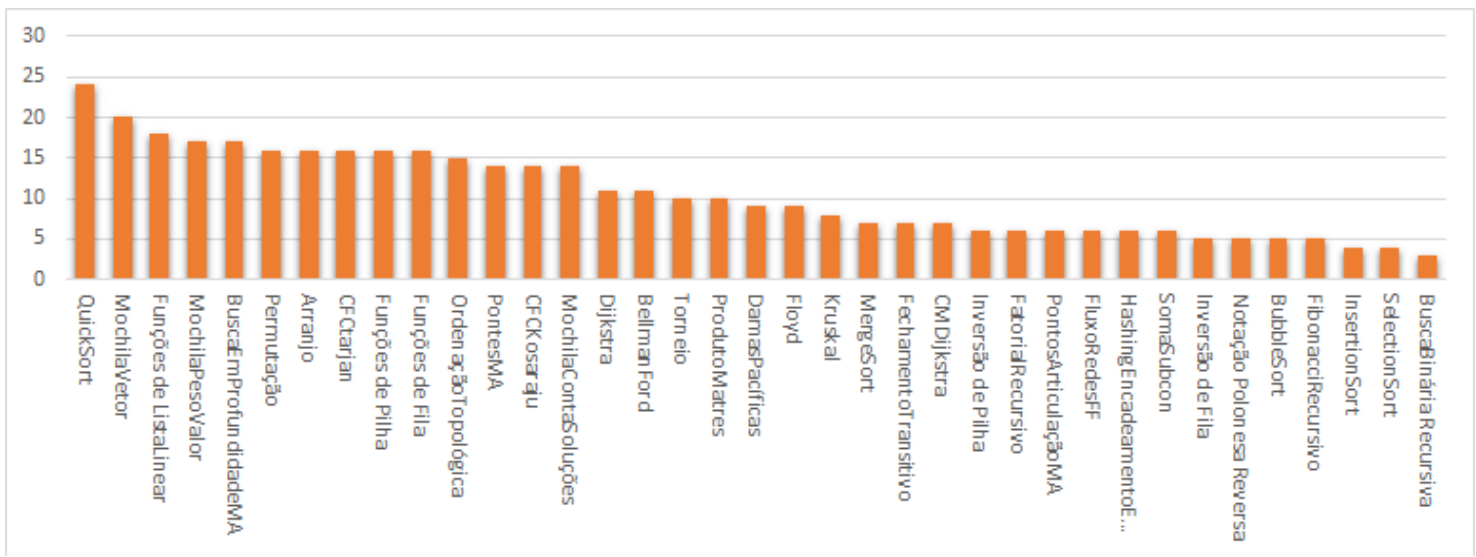

Figura 8. Programas simulados pelos alunos por ordem decrescente.

\section{Conclusão}

Neste trabalho, apresenta-se o TuPy Online, criado com o objetivo principal de suportar a visualização das estruturas de dados em alto nível de abstração. Apresentase também sua avaliação de usabilidade e sua eficiência na aprendizagem de algoritmos. Quanto à avaliação de aprendizagem, o modelo adaptado do questionário de Savi et al. para avaliação da ferramenta demonstrou alto índice de concordância dos entrevistados em relação à relevância da proposta do TuPy Online e à praticidade de sua interface. A avaliação sistemática é conclusiva em relação à influência positiva da ferramenta na aprendizagem. A auto-avaliação demonstrou a satisfação dos alunos de todas as turmas. Em relação à usabilidade, em uma primeira avaliação, o resultado foi positivo identificando possíveis melhorias e aperfeiçoamento do TuPy Online.

Além disso, o complemento da avaliação de usabilidade indicou pontos positivos e negativos para possível aprimoramento da ferramenta. A facilidade de uso, a utilização de um código em português, possibilidade de visualizações customizadas foram pontos positivos encontrados pelos alunos no TuPy Online. Em relação aos pontos negativos, os alunos indicaram falta de exemplos mais básicos no programa, falta de uma explicação mais abrangente em como customizar visualizações, problemas no enquadramento de visualizações com maior grau de complexidade, entre outros. Apesar dos pontos negativos, cabe ressaltar que todas as avaliações indicaram de maneira positiva a utilização do TuPy Online para o aprendizado de algoritmos. Resultados parciais deste trabalho foram apresentados em [Roberto et al. 2018, Goulart et al. 2019a, Goulart et al. 2019b].

Como trabalho futuro, além de melhorias identificadas pela avaliação de usabilidade, pretendemos integrar outras ferramentas, tal como a visualização de gráficos, e 
também internacionalizá-la, pelo reconhecimento de pseudocódigos em outros idiomas.

\section{Referências}

Brooke, J. (1996). SUS- A quick and dirty usability scale. Usability evaluation in industry, 189(194):4-7.

Ferreira, A. (2017). MA-AVA: Modelo de Avaliação da Aprendizagem em Ambientes Virtuais. Dissertação de Mestrado. Universidade do Estado do Rio de Janeiro.

Goulart, J., Oliveira, F., and Pinto, P. (2019a). Avaliacão Sistemática de Eficácia na Aprendizagem de Algoritmos com o uso do Tupy Online. 27 ${ }^{\circ}$ Workshop sobre Educação em Computação (WEI).

Goulart, J., Oliveira, F., Pinto, P., Roberto, G., and Sathler, V. (2019b). Tupy online: Uma ferramenta para visualização de algoritmos. In Anais dos Workshops do Congresso Brasileiro de Informática na Educação, volume 8, page 337.

Keller, J. (1983). Development and use of the ARCS model of motivational design. Technical report, Exeter, England: at the annual meeting of the Association for Education and Training Technology.

Krathwohl, D. (2002). A Revision of Bloom's Taxonomy: An Overview. Theory into practice, 41(4):212-218.

Moody, D. and Sindre, G. (2003). Evaluating the Effectiveness of Learning Interventions: An Information Systems Case Study. European Conference on Information Systems (ECIS).

Roberto, G., Oliveira, F., Pinto, P., and Coelho, I. (2018). Tupy Online - Programação em Português com Visualização de Execução e Abstrações de Estruturas de Dados na Web. $26^{\circ}$ Workshop sobre Educação em Computação (WEI).

Sauro, J. (2011). Measuring usability with the system usability scale (sus). 2011. URL: http://www. measuringusability. com/sus. php [accessed 2019-09-25].

Savi, R., Von Wangenheim, C. G., Ulbricht, V., and Vanzin, T. (2010). Proposta de um modelo de avaliação de jogos educacionais. RENOTE-Revista Novas Tecnologias na Educação, 8(3). 